\title{
Spectrum and Drug Susceptibility Profile of Bacteria Recovered from Patients with Wound Infection Referred to Arsho Advanced Medical Laboratory
}

\author{
Adane Bitew ${ }^{1, ~ *}$, Mesele Admassie ${ }^{2}$, Tigist Getachew ${ }^{1}$ \\ ${ }^{1}$ Department of Medical Laboratory Sciences, College of Health Sciences, Addis Ababa University, Addis Ababa, Ethiopia \\ ${ }^{2}$ Arsho Advanced Medical Laboratory Private Limited Company, Addis Ababa, Ethiopia \\ Email address: \\ bitewadane@gmail.com (A. Bitew), mesele2006@gmail.comm (M. Admassie), tigist.getachew@aau.edu.et (T. Getachew) \\ ${ }^{*}$ Corresponding author
}

\section{To cite this article:}

Adane Bitew, Mesele Admassie, Tigist Getachew. Spectrum and Drug Susceptibility Profile of Bacteria Recovered from Patients with wound Infection Referred to Arsho Advanced Medical Laboratory. Clinical Medicine Research. Vol. 7, No. 1, 2018, pp. 8-17.

doi: $10.11648 /$ j.cmr.20180701.12

Received: January 4, 2018; Accepted: February 9, 2018; Published: March 7, 2018

\begin{abstract}
Wound infection still remains a significant cause of morbidly and mortality. Hence, studying the spectrum of bacterial etiological agents and their drug susceptibility profile is critical. A prospective study was conducted at Arsho Advanced Medical Laboratory from June 2016 to July 2017. Wound specimens were collected from 366 patients following standard procedures. Specimens were plated and incubated at $37^{\circ} \mathrm{C}$ for 48 hours. Identification and drug susceptibility testing of cultures were carried out by using the VITEK 2 compact system. Among 366 wound samples cultured, bacteria grew in 271(74\%) samples. The highest (81.9\%) wound infections were documented among patients with an age group of 15-64 years. Two hundred twenty one bacterial isolates were recovered of which $43.2 \%$ were Gram-negative while, $56.8 \%$ were Grampositive. Staphylococcus aureus and Coagulase-Negative Staphylococci were major Gram-positive bacteria while Escherichia coli and Pseudomonas spp. were the commonest Gram-negative bacteria. Gram-negative bacteria had the highest overall drug resistance rate against ampicillin. Tobramycin and piperacillin/tazobactam combination were effective antimicrobial agents against Gram-negative bacteria. The highest overall resistance rate to Gram-positive bacteria was observed against erythromycin. Vancomycin and linezolid were the most active antimicrobial agents against Gram-positive bacteria. High culture positivity rate of wound infections reported in the present study initiates many similar studies to be conducted on wound in the country. High level of drug resistance to the commonly prescribed drugs dictates a search for better choices.
\end{abstract}

Keywords: Wound Infections, Drug Suscetibity Pattern, Etiological Agents, Ethiopia

\section{Introduction}

The human skin is one of our first line innate immunities that prevents infections of internal tissues by microorganisms physically. Sweat and sebaceous secretions produced by the skin also deny microbial infections of internal tissues by a virtue of their acidic $\mathrm{pH}$ (3-5). Moreover, fatty acids that have antifungal properties and lysozyme that dissolves bacterial cell wall also play a major role in the protection of internal tissues by microbial pathogens. Wound is, therefore, a break in the skin that exposes internal tissues to pathogens. It provides moist, warm, and conducive situation that is favorable for bacterial colonization and propagation [1]. Accidental (e.g., thermal wound infection) or intentionally (surgical or use of intravenous medical devices) induced trauma is an indispensable incident for all wound colonization. Wounds develop into an infected state when the balance between microorganism and the host shifts in favour of the micro-organism [2].

Wound infections can be classified into skin infection and soft tissue infection. Wound infections can also be classified into community acquired and hospital acquired infection [3] where the latter is one of the prominent nosocomial causes of morbidity. Hospital- acquired wound infections result in 
repeated hospitalization, lengthy hospital stay, increased demand of wound care, and treatment cost. It is also a major cause of anxiety in health workers causing wound management practices much more challenging $[4,5]$.

Bacterial etiologies of wound infections have been excellently reviewed by Howell-Jone et al [6]. Although bacterial etiologies of wound infections vary within countries and hospitals in the same country [7], S. aureus and Coagulase-Negative Staphylococci have been the major bacteria isolated irrespective of the type of study [6].

Wound infection still remains a substantial cause of morbidly and mortality particularly in developing countries, although major achievements in its control and management have been achieved [8]. This is because, wound infections are one of the major sources of post-operative disorder, that cause mortality among burn patients [9], and accounts for roughly one-fourth of all hospital acquired infections [10]. To this effect, identification and determining drug susceptibility pattern of bacteria associated with wound infections for efficient management of patients with the problem is still an active field of research. Although numerous researches have been conducted on wound infections in Ethiopia, a change in etiologic agents and poor laboratory set up coupled with the development of drug resistance warranted additional investigation.

In Ethiopia, like other developing countries, diagnostic microbiology laboratories are poorly organized. Diagnostic laboratories that isolate and characterize bacteria by using even few routine biochemical tests are rare. Furthermore, drug susceptibility testing of bacterial isolates has also been determined by using agar diffusion technique with all its limitations. Consequently, treatment of bacterial wound infection in Ethiopia has remained empirical. In addition, agreement with respect to the distribution of bacterial species associated with wound infections and their drug susceptibility pattern among different local studies is lacking. In view of this, application of fully automated systems for bacterial characterization and for the assessment of their antimicrobial susceptibility profile has become important. The VITEK 2 compact (bioMérieux, France) is a machine capable of running bacterial identification and drug susceptibility testing at the same time. Reduced turnaround times, better specimen management, enhanced quality control, reproducibility, precision, and the ability to track results are other benefits of the VITEK 2 compact system over conventional methods. With regards to Identification, the machine characterize a total of 135 Gram-negative fermenting and non-fermenting bacilli and 115 Grampositive cocci and non-spore-forming bacilli to the species level by using 64 biochemical tests and substrates. Identification of bacterial isolates to species level provides indispensable information on its pathogenic potential and is of greatest importance for the correct explanation of antibiotic susceptibility testing. Against this background, the objective of this study was to characterize and evaluate drug susceptibility profile of bacteria associated with wound infections from patients referred to Arsho Advanced Medical
Laboratory by employing the fully automated VITEK 2 compact system.

\section{Materials and Methods}

This study was carried out at Arsho Advanced Medical laboratory, Addis Ababa, Ethiopia from June 2016 to July 2017. Arsho is the oldest Medical Laboratory where patients are referred to culture and drug sensitivity testing. On the average about 50 patients per day are referred to Arsho for culture and drug sensitivity testing. It is also the only diagnostic laboratory in the country where automated machines such as the VITEK 2 compact system is employed for routine diagnostic and/or research activity. The requisition form filled out by physicians was used as standard proforma to document socio-demographic characteristics, history of antibiotic treatment and other information about study subjects. Patients to be included in the study, they must be clinically diagnosed for wound infection, consent to participate in the study, and no anti-bacterial therapy is administered within two weeks prior to their attendance.

Wound specimens were collected aseptically from study participants following standard procedures. Clinical samples such as biopsy and tissue materials collected and referred from respective health institutions were also used in this study. All wound samples were then streaked onto primary isolation culture media (Blood Agar base (Oxoid, Basingstoke, Hampaire, UK ) to which $10 \%$ sheep blood is incorporated, Mannitol salt agar(Oxoid, Basingstoke, Hampaire, UK), MacConkey agar (Oxoid, Basingstoke, Hampaire, UK), and Chocolate agar), incubated at $37^{\circ} \mathrm{C}$ for 18-24 hours aerobically. Pure isolates of significant bacterial pathogen per sample were preliminary characterized by colony morphology, Gram-stain, lactose fermentation, and catalase test before inoculating them into AST-GN72 and AST-GP71 cards.

Identification and drug sensitivity testing of pure cultures were carried by the VITEK 2 compact system following the procedures of the manufacture (bioMérieux, France). ASTGN72 cards (kits used for the identification and susceptibility testing Gram-negative bacteria) were used for the identification and susceptibility testing of fermenting and non-fermenting Gram-negative bacilli, while the AST-GP71 cards (kits used for the identification and susceptibility testing of Gram-positive bacteria) were used for the identification and susceptibility testing of non-spore-forming Gram-positive bacteria. Detailed description of inoculum size determination, bacterial identification and drug sensitivity testing by the machine, and the antimicrobial agents used with their concentration can be obtained from Bitew et al [11].

\section{Ethics and Consent to Participate}

All ethical considerations and obligations were duly addressed. The study was carried out after the approval of research and ethical committee of Arsho Advanced Medical 
Laboratory private limited company (AAMLRERC). Data collection was started after obtaining written informed consent from study subjects and assent form was completed and signed. All the information obtained from the study subjects were coded to maintain confidentially.

\section{Results}

Out of 366 wound samples studied, $153(41.8 \%)$ were collected from female and $213(58.2 \%)$ from male study subjects. Among wound samples studied, 271 (74\%) showed bacterial growth where $109(40.2 \%)$ were collected from female and $162(59.8 \%)$ from male study subjects. Two hundred twenty two $(81.9 \% ; 222 / 271)$ wound infections were documented from young and middle age patients with an age group of 15-64 years. Of a total of 271 individuals with wound infections, pediatric study subjects (0-14 years) accounted for $3.7 \%$ while elderly study subjects ( $\geq 65$ years) accounted for $14.4 \%$ (Table 1). The highest wound infection $(39.5 \%)$ was recorded in patients of age group 45-64 followed by age group of 25-44.

Table 1. Frequency of wound infection in relation to gender and age $(n=366)$.

\begin{tabular}{lllll}
\hline Variables & category & Sample size & Culture positive samples, n (\%) & Culture negative samples, n (\%) \\
\hline \multirow{3}{*}{ Gender } & Overall & 366 & $271(74)$ & $95(26)$ \\
& Female & $153(41.8)$ & $109(40.2)$ & $44(46.3)$ \\
& Male & $213(58.2)$ & $162(59.8)$ & $51(53.7)$ \\
& Total & $366(100)$ & $271(100)$ & $95(100)$ \\
& $<1$ & $4(1.1)$ & $3(1.1)$ & $7(1.0)$ \\
& $1-14$ & $14(3.8)$ & $51(18.8)$ & $7(7.4)$ \\
Age in years & $15-24$ & $68(18.6)$ & $64(23.6)$ & $17(17.9)$ \\
& $25-44$ & $86(23.5)$ & $107(39.5)$ & $22(23.2)$ \\
& $45-64$ & $145(39.6)$ & $39(14.4)$ & $38(40)$ \\
& $65+$ & $49(13.4)$ & $271(100)$ & $95(10.5)$ \\
\hline
\end{tabular}

Out of 271 bacterial isolates recovered, 117(43.2\%; 117/271) were Gram-negative while 154 (56.8\%; 154/271) were Gram-positive bacteria. S. aureus and CoagulaseNegative Staphylococci were the major Gram-positive bacteria, comprising of $(40.6 \% ; 110 / 271)$ and $12.9 \%$
$(35 / 271)$ of the total isolates, respectively. The four major genera of Gram-negative bacteria isolated include Escherichia 49 (18.1\%), Pseudomonas 15 (5.5\%), Klebsiella 14 (5.2), and Proteus 12 (4.4\%) (Tables 2 and 3).

Table 2. Distribution and percentage frequency of Gram- negative bacterial species ( $n=117)$.

\begin{tabular}{lll}
\hline Genus & Species & n (\%) of the total isolates \\
\hline Acenitobacter & A. baumanni & $4(1.5)$ \\
Burkholderai & A. calcooceticus & $2(0.7)$ \\
Citrobacter & B. cepacia & $1(0.4)$ \\
Escherichia & C. diversus & $3(1.1)$ \\
Enterobacter & C. freundii & $1(0.4)$ \\
Klebsiella & E. coli & $49(18.1)$ \\
Morganella & E.cloacae complex & $6(2.2)$ \\
Proteus & K. pneumonia & $12(4.4)$ \\
Providentia & K. oxytoca & $2(0.7)$ \\
Pseudomonas & M. morganii & $3(1.1)$ \\
& P. mirabilis & $7(2.6)$ \\
Raoultella & P.vulgaris & $5(1.8)$ \\
Salmonella & Providentia retgerii & $1(0.4)$ \\
Serratia & P. aeruginosa & $14(5.2)$ \\
Total (13) & P. luteola & $1(0.4)$ \\
& R. planticola & $2(0.74)$ \\
\hline
\end{tabular}

The overall drug susceptibility profile of Gram-positive bacteria against the sixteen antimicrobial agents evaluated is presented in Table 4. The highest overall resistance rate to Gram-positive bacteria was recorded against erythromycin $(60.4 \%)$. The resistance rates to tetracycline $(51.3 \%)$ and clindamycin $(52.2 \%)$ were also high. Vancomycin with a sensitivity rate of $99.1 \%$ and linezolid with a sensitivity rate of $98.7 \%$ were the most active drugs against Gram-positive bacteria. The sensitivity rates of $S$. aureus strains and Coagulase-Negative Staphylococci were $100 \%$ to vancomycin, linezolid, and daptomcin. 
Table 3. Distribution and percentage frequency of Gram-positive bacterial species $(n=154)$.

\begin{tabular}{lll}
\hline Genus & Species & n (\%) of the total isolates \\
\hline \multirow{2}{*}{ Enterococcus } & E. faecalis & $1(0.4)$ \\
& E.avium & $1(0.4)$ \\
Kocuria & K. intermides & $1(0.4)$ \\
Staphylococcus & K. varians & $1(0.4)$ \\
& S. aureus & $110(40.6)$ \\
& S. epidermidis & $15(5.5)$ \\
Coagulation negative staphylococci & S. haemolyticus & $9(3.3)$ \\
& S. hominis & $5(1.8)$ \\
& S. lentus & $3(1.1)$ \\
Streptococcus & S. intermidus & $2(0.7)$ \\
Total (4) & S. lugdunensis & $1(0.4)$ \\
& S. pyogens & $5(1.8)$ \\
& 13 & $154(56.8)$ \\
\hline
\end{tabular}

Table 4. Percentage in vitro antibacterial susceptibility pattern of all Gram-positive bacteria isolates $(n=154)$.

\begin{tabular}{|c|c|c|c|c|c|c|c|c|c|c|c|c|c|c|c|c|c|}
\hline \multirow{2}{*}{ Species } & \multicolumn{17}{|c|}{ Antibiotics } \\
\hline & $\mathbf{P}$ & CIP & CM & $\mathbf{E}$ & GM & LEV & LIN & MNO & MFX & FT & QDA & RA & TE & TRM & VA & TGC & DAP \\
\hline \multirow{3}{*}{ S. aureus (110) } & $\mathrm{S}$ & 91.0 & 33.6 & 22.7 & 85.5 & 80.1 & 100 & 77.2 & 83.6 & 79.1 & 91.8 & 68.2 & 17.3 & 66.4 & 99.1 & 96.4 & 98.2 \\
\hline & I & 2.7 & 9.1 & 10.9 & 12.7 & 14.5 & 0 & 6.4 & 6.4 & 9.1 & 0.9 & 9.1 & 27.3 & 10.9 & 0.9 & 2.7 & 1.8 \\
\hline & $\mathrm{R}$ & 6.3 & 57.3 & 63.4 & 1.8 & 4.5 & 0 & 16.4 & 10.0 & 11.8 & 6.3 & 31.8 & 55.5 & 27.7 & 0 & 0.9 & 0 \\
\hline \multirow{3}{*}{$\begin{array}{l}\text { S. epidermidis } \\
(15)\end{array}$} & $\mathrm{S}$ & 86.7 & 20.0 & 33.3 & 93.3 & 86.7 & 100 & 80 & 86.7 & 73.3 & 80 & 60 & 46.7 & 53.3 & 100 & 86.7 & 100 \\
\hline & I & 6.7. & 26.7 & 13.3 & 0 & 6.7 & 0 & 6.7 & 6.7 & 20.0 & 6.7 & 26.7 & 6.7 & 20.0 & 0 & 6.7 & 0 \\
\hline & $\mathrm{R}$ & 6.7 & 53.3 & 53.3 & 6.7 & 6.7 & 0 & 13.3 & 6.7 & 6.7 & 13.3 & 13.3 & 46.7 & 26.7 & 0 & 6.7 & 0 \\
\hline \multirow{2}{*}{$\begin{array}{l}\text { S. haemolyticus } \\
\text { (9) }\end{array}$} & I & 11.1 & 11.1 & 11.1 & 0 & 22.2 & 0 & 0 & 0 & 11.1 & 11.1 & 33.3 & 11.1 & 22.2 & 0 & 11.1 & 0 \\
\hline & $\mathrm{R}$ & 11.1 & 55.6 & 33.3 & 11.1 & 11.1 & 0 & 22.2 & 22.2 & 11.1 & 11.1 & 22.2 & 55.6 & 33.3 & 0 & 0 & 0 \\
\hline \multirow{3}{*}{ S. hominis (5) } & $\mathrm{S}$ & 40.0 & 40.0 & 40.0 & 100 & 60 & 100 & 80 & 80 & 80 & 80 & 60 & 40 & 60 & 100 & 80 & 100 \\
\hline & I & 0 & 0 & 0 & 0 & 20 & 0 & 20 & 0 & 0 & 0 & 0 & 20 & 20 & 0 & 0 & 0 \\
\hline & $\mathrm{R}$ & 60.0 & 60.0 & 60.0 & 0 & 20 & 0 & 0 & 20 & 20 & 20 & 40 & 40 & 20 & 0 & 20 & 0 \\
\hline \multirow[b]{2}{*}{ S. lentus (3) } & $\mathrm{S}$ & 66.7 & 33.3 & 33.3 & 66.7 & 66.7 & 100 & 67.3 & 100 & 100 & 33.3 & 33.3 & 33.3 & 66.7 & 100 & 66.7 & 100 \\
\hline & I & 33.3 & 0 & 33.3 & 33.3 & 33.3 & 0 & 33.3 & 0 & 0 & 33.3 & 33.3 & 33.3 & 0 & 0 & 33.3 & 0 \\
\hline \multirow{3}{*}{ S. intermidus (2) } & $\mathrm{S}$ & 50 & 50.0 & 50.0 & 100 & 50 & 100 & 50 & 100 & 100 & 50 & 0 & 100 & 50 & 100 & 100 & 100 \\
\hline & I & 0 & 50.0 & 0 & 0 & 50 & 0 & 50 & 0 & 0 & 0 & 50 & 0 & 0 & 0 & 0 & 0 \\
\hline & $\mathrm{R}$ & 50 & 0 & 50.0 & 0 & 0 & 0 & 0 & 0 & 0 & 50 & 50 & 0 & 50 & 0 & 0 & 0 \\
\hline \multirow{3}{*}{ S. lugdunensis (1) } & $\mathrm{S}$ & 0 & 0 & 0 & 100 & 100 & 100 & 100 & 0 & 0 & 100 & 100 & 100 & 0 & 100 & 100 & 100 \\
\hline & I & 0 & 0 & 0 & 0 & 0 & 0 & 0 & 100 & 100 & 0 & 0 & 0 & 100 & 0 & 0 & 0 \\
\hline & $\mathrm{R}$ & 100 & 100 & 100 & 0 & 0 & 0 & 0 & 0 & 0 & 0 & 0 & 0 & 0 & 0 & 0 & 0 \\
\hline \multirow{3}{*}{ S. pyogens (5) } & $\mathrm{S}$ & 80 & 60.0 & 40 & 60 & 80 & 100 & 80 & 40 & 40 & 80 & 40 & 20 & 20 & 100 & 100 & 100 \\
\hline & I & 0 & 0 & 20 & 20 & 0 & 0 & 0 & 60 & 20 & 0 & 20 & 40 & 60 & 0 & 0 & 0 \\
\hline & $\mathrm{R}$ & 20 & 40.0 & 40 & 20 & 20 & 0 & 20 & 0 & 20 & 20 & 40 & 40 & 20 & 0 & 0 & 0 \\
\hline
\end{tabular}

Table 4. Cont'D

\begin{tabular}{|c|c|c|c|c|c|c|c|c|c|c|c|c|c|c|c|c|c|}
\hline \multirow{2}{*}{ Species } & \multicolumn{17}{|c|}{ Antibiotics } \\
\hline & $\mathbf{P}$ & CIP & $\mathbf{C M}$ & $\mathbf{E}$ & GM & LEV & LIN & MNO & MFX & FT & QDA & $\mathbf{R A}$ & TE & TRM & VA & TGC & DAP \\
\hline \multirow{3}{*}{ E. faecalis (1) } & $\mathrm{S}$ & 100 & 0 & 0 & 0 & 100 & 100 & 0 & 0 & 100 & 100 & 0 & 100 & 0 & 100 & 0 & 100 \\
\hline & I & 0 & 100 & 100 & 100 & 0 & 0 & 0 & 100 & 0 & 0 & 100 & 0 & 100 & 0 & 100 & 0 \\
\hline & $\mathrm{R}$ & 0 & 0 & 0 & 0 & 0 & 0 & 100 & 0 & 0 & 0 & 0 & 0 & 0 & 0 & 0 & 0 \\
\hline \multirow{3}{*}{ E. avium (1) } & $\mathrm{S}$ & 100 & 0 & 100 & 0 & 100 & 100 & 0 & 0 & 100 & 0 & 100 & 100 & 0 & 100 & 0 & 100 \\
\hline & I & 0 & 100 & 0 & 100 & 0 & 0 & 0 & 100 & 0 & 0 & 0 & 0 & 100 & 0 & 100 & 0 \\
\hline & $\mathrm{R}$ & 0 & 0 & 0 & 0 & 0 & 0 & 100 & 0 & 0 & 100 & 0 & 0 & 0 & 0 & 0 & 0 \\
\hline \multirow{2}{*}{$\begin{array}{l}\text { Kocuria intermides } \\
\text { (1) }\end{array}$} & I & 0 & 100 & 100 & 0 & 0 & 100 & 100 & 100 & 0 & 100 & 100 & 0 & 0 & 100 & 0 & 0 \\
\hline & $\mathrm{R}$ & 0 & 0 & 0 & 0 & 0 & 0 & 0 & 0 & 0 & 0 & 0 & 100 & 0 & 0 & 100 & 0 \\
\hline \multirow{3}{*}{ K. varians $(1)$} & $\mathrm{S}$ & 100 & 0 & 0 & 100 & 100 & 0 & 0 & 0 & 100 & 0 & 0 & 0 & 100 & 100 & 100 & 0 \\
\hline & I & 0 & 100 & 100 & 0 & 0 & 100 & 100 & 100 & 0 & 100 & 100 & 100 & 0 & 0 & 0 & 100 \\
\hline & $\mathrm{R}$ & 0 & 0 & 0 & 0 & 0 & 0 & 0 & 0 & 0 & 0 & 0 & 0 & 0 & 0 & 0 & 0 \\
\hline \multirow[b]{2}{*}{ Total 154} & $\mathrm{~S}$ & 86.4 & 31.2 & 27.3 & 85.1 & 77.9 & 98.7 & 75.3 & 80.5 & 79.2 & 85.7 & 61.7 & 24.7 & 61.0 & 99.1 & 92.2 & 98.7 \\
\hline & I & 4.5 & 13.6 & 13.3 & 11.7 & 14.3 & 1.3 & 8.4 & 10.0 & 10.4 & 4.5 & 24.0 & 24.0 & 15.6 & 0.9 & 5.2 & 1.3 \\
\hline
\end{tabular}

$\mathrm{CIP}=$ ciprofloxacin, $\mathrm{CM}=$ clindamycin. $\mathrm{E}=$ erythromycin, $\mathrm{GM}=$ gentamicin, $\mathrm{LEV}=$ Levofloxacin, $\mathrm{LIN}=\mathrm{Linezolid}, \mathrm{MNO}=$ minocycline, $\mathrm{MFX}=$ moxifloxacine, $\mathrm{FT}=$ nitrofurantoin, $\mathrm{QDA}=$ quinupristin/dalfopristin, $\mathrm{RA}=$ rifampicin, $\mathrm{TE}=$ tetracycline, $\mathrm{TRM}=$ trimethoprim/sulfamethoxazole, $\mathrm{VA}=\mathrm{vancomicin}, \mathrm{TGC}-$ Tigecyline, $\mathrm{DAP}=$ daptomycin, $\mathrm{S}=$ Sensitive, $\mathrm{I}=$ Intermediate, $\mathrm{R}=$ Resistance, $\mathrm{P}=\mathrm{Pattern}$. 
Table 5. Percentage in vitro antibacterial susceptibility pattern of all Gram-negative bacteria isolates $(n=117)$.

\begin{tabular}{|c|c|c|c|c|c|c|c|c|c|c|c|c|c|c|c|c|c|c|c|c|}
\hline \multirow{2}{*}{ Species } & \multicolumn{20}{|c|}{ Antibiotics } \\
\hline & $\mathbf{P}$ & $\mathbf{A M}$ & AMC & CIP & $\mathrm{CZ}$ & CXM & CXMA & FOX & CF & CPD & CAZ & CRO & FEP & GM & LEV & FT & TZP & TM & TE & SXT \\
\hline \multirow{3}{*}{ E. Coli (49) } & $\mathrm{S}$ & 16.3 & 18.4 & 77.6 & 40.8 & 34.7 & 38.8 & 30.6 & 16.3 & 28.6 & 71.4 & 65.3 & 67.3 & 73.5 & 61.2 & 91.8 & 93.9 & 91.8 & 28.5 & 34.7 \\
\hline & I & 12.2 & 10.2 & 12.2 & 36.7 & 26.5 & 40.8 & 12.2 & 40.8 & 8.2 & 12.2 & 14.3 & 4.1 & 16.3 & 20.4 & 2.0 & 2.0 & 4.1 & 24.5 & 20.4 \\
\hline & $\mathrm{R}$ & 71.4 & 71.4 & 10.2 & 22.4 & 38.8 & 20.4 & 64.3 & 42.9 & 4.1 & 16.3 & 20.4 & 28.6 & 10.2 & 38.8 & 6.1 & 4.1 & 4.1 & 46.9 & 44.9 \\
\hline \multirow{3}{*}{$\begin{array}{l}K . \\
\text { pneumonia } \\
(12)\end{array}$} & $\mathrm{S}$ & 0 & 0 & 75 & 16.7 & 25 & 33.3 & 41.7 & 25 & 16.7 & 66.7 & 41.7 & 33.3 & 66.7 & 75 & 58.3 & 91.7 & 83.3 & 16.7 & 33.3 \\
\hline & I & 0 & 0 & 8.3 & 16.7 & 16.7 & 8.3 & 8.3 & 25 & 8.3 & 8.3 & 0.0 & 0 & 16.7 & 16.7 & 16.7 & 0 & 8.3 & 16.7 & 25 \\
\hline & $\mathrm{R}$ & 100 & 100 & 16.7 & 66.7 & 58.3 & 58.3 & 50.0 & 50 & 75.0 & 25.0 & 58.3 & 66.7 & 16.7 & 8.3 & 25.0 & 8.3 & 8.3 & 67.7 & 41.7 \\
\hline \multirow{3}{*}{$\begin{array}{l}P . \\
\text { aeruginosa } \\
\text { ( } 14)\end{array}$} & S & 0 & 0 & 85.7 & 21.4 & 14.3 & 7.1 & 7.1 & 14.3 & 7.1 & 64.3 & 21.4 & 21.4 & 85.7 & 64.3 & 57.1 & 85.7 & 85.7 & 0 & 21.4 \\
\hline & I & 0 & 0 & 7.1 & 28.6 & 14.3 & 14.3 & 7.1 & 42.9 & 7.1 & 7.1 & 0 & 7.1 & 7.1 & 14.3 & 7.1 & 7.1 & 7.1 & 7.1 & 14.3 \\
\hline & $\mathrm{R}$ & 100 & 100 & 7.1 & 50 & 71.4 & 78.6 & 85.7 & 42.9 & 85.7 & 28.6 & 78.6 & 71.4 & 7.1 & 21.4 & 35.7 & 7.1 & 7.1 & 92.9 & 64.3 \\
\hline \multirow{3}{*}{$\begin{array}{l}\text { E.cloacae } \\
\text { complex }(6)\end{array}$} & S & 0 & 0 & 66.7 & 16.7 & 33.3 & 33.3 & 16.7 & 33.3 & 33.3 & 66.7 & 50 & 33.3 & 66.7 & 66.7 & 66.7 & 66.7 & 66.7 & 16.7 & 33.3 \\
\hline & I & 33.3 & 50 & 0 & 50 & 33.3 & 33.3 & 16.7 & 16.7 & 16.7 & 33.3 & 33.3 & 33.3 & 0 & 16.7 & 0 & 16.7 & 16.7 & 16.7 & 33.3 \\
\hline & $\mathrm{R}$ & 66.7 & 50 & 33.3 & 33.3 & 33.3 & 33.3 & 66.7 & 50 & 50.0 & 0 & 16.7 & 33.3 & 33.3 & 16.7 & 33.3 & 16.7 & 16.7 & 66.7 & 33.3 \\
\hline \multirow{3}{*}{$\begin{array}{l}\text { P. mirabilis } \\
\text { (7) }\end{array}$} & $\mathrm{S}$ & 14.3 & 14.3 & 71.4 & 57.1 & 57.1 & 57.1 & 28.6 & 42.9 & 28.6 & 100 & 28.6 & 42.9 & 71.4 & 71.4 & 85.7 & 87.5 & 100 & 14.3 & 28.6 \\
\hline & I & 14.3 & 28.6 & 0 & 28.6 & 14.3 & 42.9 & 14.3 & 14.3 & 14.3 & 0 & 0 & 0 & 0 & 14.3 & 14.3 & 0 & 0 & 0 & 14.3 \\
\hline & $\mathrm{R}$ & 71.4 & 57.1 & 28.6 & 14.3 & 28.6 & 0 & 57.1 & 42.9 & 57.1 & 0 & 71.4 & 57.1 & 28.6 & 14.3 & 0 & 14.3 & 0 & 85.7 & 67.0 \\
\hline \multirow{3}{*}{$\begin{array}{l}\text { P. vulgaris } \\
\text { (5) }\end{array}$} & $\mathrm{S}$ & 0 & 0 & 60 & 0 & 0 & 0 & 60 & 20 & 20.0 & 80 & 40.0 & 40 & 100 & 80 & 20 & 80 & 80 & 0 & 40.0 \\
\hline & I & 40 & 60 & 20 & 40 & 40 & 40 & 20 & 40 & 20 & 0 & 0 & 20 & 0 & 20 & 40 & 0 & 20 & 40 & 0 \\
\hline & $\mathrm{R}$ & 60 & 40 & 20 & 60 & 60 & 60 & 20 & 20 & 60 & 20 & 60.0 & 40 & 0 & 0 & 40 & 20 & 0 & 60 & 60.0 \\
\hline \multirow{3}{*}{$\begin{array}{l}\text { A. baumanni } \\
\text { (4) }\end{array}$} & $\mathrm{S}$ & 0 & 0 & 25 & 0 & 0 & 0 & 0 & 0 & 0 & 25 & 25 & 25 & 25 & 50 & 0 & 25 & 50 & 0 & 0 \\
\hline & I & 0 & 0 & 25 & 0 & 0 & 0 & 0 & 0 & 0 & 0 & 25 & 25 & 0 & 25 & 25 & 50 & 50 & 0 & 0 \\
\hline & $\mathrm{R}$ & 100 & 100 & 50 & 100 & 100 & 100 & 100 & 100 & 100 & 75 & 50 & 50 & 75 & 25 & 75 & 25 & 0 & 100 & 100 \\
\hline \multirow{3}{*}{$\begin{array}{l}\text { M. morganii } \\
\text { (3) }\end{array}$} & $\mathrm{S}$ & 0 & 0 & 66.7 & 0 & 0 & 0 & 66.7 & 33.3 & 100 & 100 & 0 & 100 & 66.7 & 100 & 66.7 & 66.7 & 100 & 0 & 33.3 \\
\hline & I & 0 & 33.3 & 33.3 & 0 & 0 & 0 & 33.3 & 33.3 & 0 & 0 & 100 & 0 & 0 & 0 & 0 & 33.3 & 0 & 0 & 66.7 \\
\hline & $\mathrm{R}$ & 100 & 66.7 & 0 & 100 & 100 & 100 & 0 & 33.3 & 0 & 0 & 0 & 0 & 33.3 & 0 & 33.3 & 0 & 0 & 100 & 0 \\
\hline
\end{tabular}

Table 5. Cont' $D$.

Species

\section{Antibiotics}

$\begin{array}{lllllllllllllllll}P & \text { AM } & \text { AMC } & \text { CIP } & \text { CZ } & \text { CXM } & \text { CXMA FOX CF } & \text { CPD } & \text { CAZ } & \text { CRO FEP } & \text { GM } & \text { LEV } & \text { FT } & \text { TZP } & \text { TM } & \text { TE } & \text { SXT }\end{array}$

K. oxytoca (2)

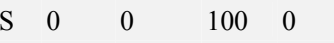

$\begin{array}{lllllll}100 & 100 & 50 & 100 & 100 & 50\end{array}$

$\mathrm{R} \quad 100 \quad 50 \quad 0 \quad 100 \quad 100 \quad 100$

$\begin{array}{llllllllllllllllllllll}\text { A. calcooceticus } & \mathrm{S} & 0 & 0 & 0 & 0 & 0 & 0 & 0 & 0 & 0 & 0 & 0 & 0 & 50 & 0 & 0 & 50 & 100 & 0 & 50 \\ \text { (2) } & \mathrm{I} & 0 & 0 & 50 & 0 & 0 & 0 & 50 & 0 & 50 & 50 & 0 & 50 & 50 & 100 & 0 & 50 & 0 & 0 & 50\end{array}$ $\begin{array}{lllllllllllllllllllllll}\mathrm{R} & 100 & 100 & 50 & 100 & 100 & 100 & 50 & 100 & 50 & 50 & 100 & 50 & 0 & 0 & 100 & 1 & 0 & 100 & 0\end{array}$ $\begin{array}{llllllllllllllllllll}\mathrm{S} & 0 & 0 & 100 & 0 & 0 & 0 & 0 & 0 & 0 & 100 & 100 & 100 & 100 & 100 & 0 & 0 & 100 & 0 & 100\end{array}$

$\begin{array}{lllllllllllllllllllllll}\text { S. marcescens (1) I } & 0 & 0 & 0 & 0 & 0 & 0 & 100 & 0 & 100 & 0 & 0 & 0 & 0 & 0 & 0 & 100 & 0 & 0 & 0\end{array}$ $\begin{array}{lllllllllllllllllllll}\mathrm{R} & 100 & 100 & 0 & 100 & 100 & 100 & 0 & 100 & 0 & 0 & 0 & 0 & 0 & 0 & 100 & 0 & 0 & 100 & 0\end{array}$ $\begin{array}{rllllllllllllllllllll}\mathrm{S} & 0 & 0 & 50 & 0 & 0 & 0 & 0 & 0 & 50 & 50 & 50 & 50 & 50 & 50 & 0 & 50 & 50 & 0 & 50\end{array}$ (2) $\quad \begin{array}{lllllllllllllllllllllll} & \mathrm{I} & 0 & 0 & 0 & 0 & 0 & 0 & 50 & 0 & 0 & 0 & 0 & 0 & 0 & 0 & 100 & 50 & 0 & 0 & 50\end{array}$ $\begin{array}{llllllllllllllllllllll}\mathrm{R} & 100 & 100 & 50 & 100 & 100 & 100 & 50 & 100 & 50 & 50 & 50 & 50 & 50 & 50 & 0 & 0 & 50 & 100 & 0 \\ \mathrm{~S} & 0 & 0 & 100 & 0 & 0 & 0 & 50 & 0 & 100 & 100 & 50 & 100 & 100 & 100 & 50 & 100 & 100 & 0 & 0\end{array}$ $\begin{array}{lllllllllllllllllllll}R \text { planticola (2) } & \mathrm{I} & 0 & 0 & 0 & 0 & 0 & 0 & 0 & 0 & 0 & 0 & 0 & 0 & 0 & 50 & 0 & 0 & 0 & 50\end{array}$ $\begin{array}{llllllllllllllllllllll}\mathrm{R} & 100 & 100 & 0 & 100 & 100 & 100 & 50 & 100 & 0 & 0 & 50 & 0 & 0 & 0 & 0 & 0 & 0 & 100 & 50\end{array}$ $\begin{array}{lllllllllllllllllllllll} & \mathrm{S} & 0 & 0 & 0 & 0 & 0 & 0 & 0 & 0 & 0 & 0 & 0 & 0 & 0 & 0 & 0 & 0 & 0 & 0 & 0 \\ \text { B. cepacia (1) } & \mathrm{I} & 0 & 0 & 0 & 0 & 0 & 0 & 0 & 0 & 0 & 0 & 0 & 0 & 0 & 0 & 0 & 0 & 0 & 0 & 0\end{array}$ $\begin{array}{lllllllllllllllllllll}\mathrm{R} & 100 & 100 & 100 & 100 & 100 & 100 & 100 & 100 & 100 & 100 & 100 & 100 & 100 & 100 & 100 & 100 & 100 & 100 & 100\end{array}$ $\begin{array}{lllllllllllllllllllllll}\mathrm{S} & 0 & 0 & 100 & 0 & 0 & 0 & 0 & 0 & 100 & 100 & 100 & 0 & 100 & 100 & 0 & 0 & 0 & 0 & 0\end{array}$ $\begin{array}{llllllllllllllllllllll}\text { C. diversus (3) } & \mathrm{I} & 0 & 0 & 0 & 0 & 0 & 0 & 100 & 100 & 0 & 0 & 0 & 100 & 0 & 0 & 100 & 100 & 100 & 0 & 100\end{array}$ $\begin{array}{llllllllllllllllllllllll}\mathrm{R} & 100 & 100 & 0 & 100 & 100 & 100 & 0 & 0 & 0 & 0 & 0 & 0 & 0 & 0 & 0 & 0 & 0 & 100 & 0\end{array}$ $\begin{array}{lllllllllllllllllllll}\mathrm{S} & 0 & 0 & 100 & 0 & 0 & 0 & 0 & 0 & 100 & 100 & 0 & 100 & 100 & 100 & 100 & 0 & 100 & 0 & 100\end{array}$ \begin{tabular}{llllllllllllllllllllllll} 
C. freundii (1) & $\mathrm{I}$ & 0 & 0 & 0 & 0 & 0 & 0 & 0 & 0 & 0 & 0 & 0 & 0 & 0 & 0 & 0 & 100 & 0 & 0 & 0 & 0 \\
& $\mathrm{R}$ & 100 & 100 & 0 & 100 & 100 & 100 & 100 & 100 & 0 & 0 & 100 & 0 & 0 & 0 & 0 & 0 & 0 & 100 & 0 & 0 \\
\hline
\end{tabular} 
Table 5. Cont'D

\begin{tabular}{|c|c|c|c|c|c|c|c|c|c|c|c|c|c|c|c|c|c|c|c|c|}
\hline \multirow{2}{*}{ Species } & \multicolumn{20}{|c|}{ Antibiotics } \\
\hline & $\mathbf{P}$ & $\mathbf{A M}$ & AMC & CIP & $\mathrm{CZ}$ & CXM & СХМА & FOX & CF & CPD & CAZ & CRO & FEP & GM & LEV & FT & TZP & TM & TE & SXT \\
\hline \multirow{3}{*}{$\begin{array}{l}\text { Providentia } \\
\text { retgerii (1) }\end{array}$} & $\mathrm{S}$ & 0 & 0 & 0 & 0 & 0 & 0 & 100 & 0 & 100 & 100 & 0 & 0 & 0 & 100 & 0 & 0 & 0 & 0 & 0 \\
\hline & I & 0 & 0 & 0 & 0 & 0 & 0 & 0 & 0 & 0 & 0 & 0 & 100 & 0 & 0 & 100 & 100 & 100 & 0 & 100 \\
\hline & $\mathrm{R}$ & 100 & 100 & 100 & 100 & 100 & 100 & 0 & 100 & 0 & 0 & 100 & 0 & 100 & 0 & 0 & 0 & 0 & 100 & 0 \\
\hline \multirow{3}{*}{ P. luteola (1) } & $\mathrm{S}$ & 0 & 0 & 0 & 0 & 0 & 0 & 100 & 0 & 0 & 0 & 0 & 0 & 0 & 0 & 0 & 100 & 0 & 0 & 0 \\
\hline & I & 0 & 0 & 100 & 0 & 0 & 100 & 0 & 0 & 100 & 100 & 100 & 100 & 100 & 100 & 100 & 0 & 100 & 0 & 100 \\
\hline & $\mathrm{R}$ & 100 & 100 & 0 & 100 & 100 & 0 & 0 & 100 & 0 & 0 & 0 & 0 & 0 & 0 & 0 & 0 & 0 & 100 & 0 \\
\hline \multirow[t]{3}{*}{ S. enterica (1) } & I & 0 & 0 & 100 & 100 & 0 & 0 & 100 & 0 & 100 & 100 & 100 & 0 & 100 & 100 & 100 & 100 & 100 & & 100 \\
\hline & $\mathrm{R}$ & 100 & 100 & 0 & 0 & 100 & 100 & 0 & 100 & 0 & 0 & 0 & 0 & 0 & 0 & 0 & 0 & 0 & 100 & 0 \\
\hline & $\mathrm{S}$ & 7.7 & 8.5 & 63.2 & 16.2 & 24.8 & 26.5 & 25.6 & 17.1 & 26.5 & 69.2 & 41.0 & 56.4 & 72.6 & 77.8 & 64.1 & 79.5 & 83.8 & 15.4 & 29.9 \\
\hline \multirow[t]{2}{*}{ Total (117) } & I & 9.4 & 11.1 & 9.4 & 20.5 & 19.7 & 26.5 & 13.7 & 31.6 & 9.4 & 8.5 & 14.5 & 9.4 & 10.3 & 11.1 & 13.7 & 13.7 & 10.3 & 15.4 & 23.1 \\
\hline & $\mathrm{R}$ & 82.9 & 80.3 & 27.3 & 63.2 & 55.6 & 47.0 & 60.7 & 51.3 & 64.1 & 22.2 & 44.4 & 34.2 & 17.1 & 11.1 & 22.2 & 6.8 & 5.9 & 69.2 & 47.0 \\
\hline
\end{tabular}

$\mathrm{AM}=$ ampicillin, $\mathrm{AMC}=$ amoxicillin/clavulanic $\mathrm{Acid}, \mathrm{CIP}=$ ciprofloxacin, $\mathrm{CZ}=$ cefazolin, $\mathrm{CXM}=$ cefuroxime, $\mathrm{CXMA}=$ cefuroxime axetil, $\mathrm{FOX}=$ cefoxitin, $\mathrm{CF}=$ cefalotin, $\mathrm{CPD}=$ cefpodoxime, $\mathrm{CAZ}=$ ceftazidime, $\mathrm{CRO}=$ ceftriaxone, $\mathrm{FEP}=$ cefepime, $\mathrm{GM}=$ gentamicin, $\mathrm{LEV}=$ levofloxacin, $\mathrm{FT}=$ nitrofurantoin, $\mathrm{TZP}=$ piperacillin/tazobactam, $\mathrm{TM}=$ tobramaycin, $\mathrm{TE}=$ tetracycline, $\mathrm{SXT}=$ trimethoprim/sulfamethoxazole, $\mathrm{S}=\mathrm{Sensitive}, \mathrm{I}=\mathrm{Intermediate}, \mathrm{R}=\mathrm{Resistance}, \mathrm{P}=\mathrm{Pattern}$

The overall antimicrobial sensitivity pattern of Gramnegative bacteria against the nineteen agents evaluated is illustrated in Table 5. The antimicrobial resistant rates of Gram-negative bacteria in their descending order were $82.9 \%$ to ampicillin, $80.3 \%$ to amoxicillin, and (69.2\%) to tetracycline. Tobramycin and piperacillin/tazobactam with the overall resistance rates of $4.3 \%$ and $6.8 \%$, respectively were better active against Gram-negative bacteria. E. coli had equal resistance rates of $71.4 \%$ to both ampicillin and amoxicillin while the resistance rate of the bacterium to tetracycline was $\quad 46.9 \%$. Piperacillin/tazobactam, nitrofurantoin, and tobramycin were better active against $E$. coli. P. aeruginosa exhibited resistance rates of $100 \%$ to ampicillin and amoxicillin/clavulanic acid combination. The least resistance rates of the bacterium $(7.1 \%)$ was observed against Piperacillin/tazobactam tobramycin, gentamycin, and ciprofloxacin. K pneumonia showed a resistance rate of $100 \%$ for ampicillin and amoxicillin/clavulanic acid combination. The drug susceptibility rates of Acinetobacter baumanni were $\leq 50 \%$ and the bacterium was $100 \%$ resistant to ten drugs out of the nineteen drugs tested.

\section{Discussion}

Out of 366 wound specimens collected from study subjects, bacterial colonies were observed in 271 giving a culture positivity rate of $74 \%$. The culture positivity rate of wound infections in the present study was relatively higher than the culture positivity rates reported in similar studies [12-15]. However, it was lower than the culture positivity rate $(87.3 \%)$ reported by Mohammedaman et al [16]. Studies conducted in Ethiopia reported culture positivity rates of wound infections in the range of $52 \%$ to $87.3 \%$. Differences in the nature and site of wound infections may explain disparities in the culture positivity rates of wound infections in the present and earlier studies.

The spectrum and the relative frequencies of bacteria implicated in causing wound infections vary greatly among studies. In this study, $S$. aureus and E. coli were the major bacteria associated with wound infection. $S$. aureus and $E$. coli as main isolates have been reported by Mulu et al [12], Mohammedaman et al [16,] and Mulugeta et al [13]. Oladeinde et al [15] and Giacometti et al [17], however, reported $S$. aureus and $P$. aeruginosa as the commonest bacterial isolates. E. coli as a third major isolate following $S$. aureus and $P$. aeruginsa has been documented by Oladeinde et al [15] and Giacometti et al (17). In the present study, Coagulase-Negative Staphylococci were the third most frequent isolated organisms following $S$. aureus and $E$. coli. Our finding was consistent with the finding of Howell-Jones et al [6]. Cross contamination of wound from nasal colonization by $S$. aureus could be one possible explanation for high isolation rate of $S$. aureus. In the present study Coagulase Negative Staphylococci were the third dominant isolates accounting for $12.9 \%$ of the total isolates. This is predictable because Coagulase Negative Staphylococci are the dominant bacteria in our skin. A breach of skin, the physical barrier, may facilitate colonization of the human skin with Coagulase Negative Staphylococci.

Although the isolation of major predictable Gram-negative and Gram-positive bacterial pathogens from wound infections in the present study was consistent with most similar studies conducted locally $[12,13,16]$, there were some striking differences. Isolation of 34 bacterial species belonging to 17 genera in the present study was higher than the previous studies. Furthermore, their studies apparently could not isolate bacteria such as $P$. aeruginosa, P. luteola. A. baumanni, A. calcooceticus, Burkholderai cepacia, Raoultella planticola, R. ornithinolytica, Kocuria intermides and $K$. varians that made $10.2 \%$ of bacterial isolates in our study. The isolation of more bacterial species in the present study could be explained with caution by the fact that, bacteria that were not commonly isolated from wound infections may replace the ones that have been commonly isolated from wound infections under selective pressure of drugs that might have emanated from the current empirical based treatment of wound infection in Ethiopia. Isolation of P. aeruginosa, P. luteola A. baumanni, A. calcooceticus, B. cepacia, $R$. planticola, $R$. ornithinolytica $K$. intermides and K.varians that were $100 \%$ resistant for six to nineteen drugs 
may support our suggestion. Secondly, in recent years, non-fermenting Gram negative bacilli, in particular $P$. aeruginosa and $A$. baumannii, have been associated with opportunistic nosocomial infections, wound being one of the main sites of infection [18]. Application of automated method of identification (the VITEK 2 compact system) in this study could be another explanation for an increase in the diversity of bacterial isolates as identification of these bacteria by routine biochemical methods is unsatisfactory.

Identification of bacterial pathogens down to a species level is important because(a) different species have different antibiotic susceptibilities (b) serious bacterial infections caused by predictable "pathogens" have decreased in recent years in proportion to those caused by opportunistic bacteria that once were considered to be of low virulence (i.e. the incidence of opportunist infections is increasing) and (c) such infections cannot be traced epidemiologically or documented without identification of bacteria to a species level. In line with this, identification of all bacterial isolates to the species level including staphylococci that were grouped as Coagulase Negative staphylococci was another striking difference in the present study and earlier local $[12,13,16]$ and international studies $[6,14,15]$.

In this study, would infection rate was more in male patients than female patients. Furthermore, the present study revealed that the proportion of wound infections was the highest in age groups of 45-64 years. Underling diseases such as diabetes mellitus, obesity, and a decrease in immune system at advanced age could be possible explanation for the situation.

The overall drug resistance rates to Gram-negative bacterial isolates ranged from $4.3 \%$ for tobramycin to $82.9 \%$ for ampicillin. The resistance rates to amoxicillin and tetracycline were also high. The highest overall resistance rate to Gram-positive bacteria was observed against erythromycin $(60.4 \%)$, followed by tetracycline $(51.3 \%)$, and clindamycin $(52.2 \%)$. This may demonstrate that old generation antimicrobial agents (ampicillin, amoxicillin, tetracycline, erythromycin, clindamycin etc.,) as a single agent for empirical treatment of wound infections would not cover the majority of wounds infected by Gram-negative and gram positive bacteria in the study area. High level of drug resistance to the old generation antimicrobial agents in the present study was compatible with the results of similar studies conducted locally [13] and internationally [19, 20]. Availability of these anti-microbial agents without prescription and inappropriate dosing schedules may explain the isolation of high level of drug resistance against these drugs.

A notable observation was that the majority of Gramnegative bacterial isolates were more sensitive towards tobramycin and piperacillin/tazobctam combinations. Both antimicrobial agents were the most effective agents against $E$. coli, the most frequently isolated Gram-negative bacterium. Our finding was similar to the result described by Manikandan and Amsath [21] and Lu et al [22]. Bours et al [23] reported that $93 \%$ E. coli were susceptible to nitrofurantion. Our result was in line with their finding as $91 \%$ E. coli were susceptible to this antimicrobial agent. Of the nine cephalosporins tested, except the extended $\beta$ - lactam cephalosporins, the resistance rates of $E$. coli to the first and second generation of cephalosporins was very high. Our result was comparable to the reports of Manikandan and Amsath [21] and Lu et al [22].

K. pneumonia, the third most commonly isolated Gramnegative bacterium was sensitive to tobramycin, Piperacillin/tazobam combination, and fuoroquinolones (ciprofloxacin levofloxacin) as seen in other studies [22, 24]. However, eight drugs tested against the isolate failed to achieve a sensitivity rate above $35 \%$. The two extended $\beta$ lactam cephalosporins were relatively better active against the bacterium and our result was comparable to that of $\mathrm{Lu}$ et al [22], Mojtahedzadeh [24], and Juyal et al [25]. A higher sensitivity rates of the bacterium against the two extended $\beta$ lactam cephalosporins (cefepime and ceftazidime) than ours was reported by Manikandan and Amsath [21]. On the other hand Mojtahedzadeh et al [24] revealed that greater than 90\% of the isolates were resistant to ciprofloxacin, ceftazidime, cefepime and ceftriaxone.

Other entrobacteria isolated in the present study such as $P$. mirabilis P. vulgaris, M. morganii, S. marcescens, and Citrobacter species were also susceptible to tobramycin and piperacillin/tazobctam combinations. However, their sensitivity towards the old generation antimicrobial agents was high as seen in another local study [13]. E. cloacae complex on the other hand was relatively resistant to most antimicrobial agents tested against Gram- negative bacteria particularly of the first and second generation cephlosporins. Our result in this regard was comparable to Lu et al [22].

The sensitivity rates of thirteen drugs tested against $P$. aeruginosa, the second most common isolate were below $25 \%$. However, the sensitivity rates of the isolate against ciprofloxacin, gentamycin, tobramycin, and Piperacillin/tazobctam were above $80 \%$. Our result was comparable to the results of earlier studies $[15,20,21]$. Among cephalosporins tested, the two extended $\beta$-lactams (cefepime and ceftazidime) were better active against the pathogen as seen in a study conducted by $\mathrm{Lu}$ et al [22] but contrary to the findings of Mojtahedzadeh et al [24]. Mojtahedzadeh et al [24] revealed that $P$. aeruginosa was $100 \%$ resistant to cefepime, ceftazidime, ceftriaxone and ciprofloxacin. A non- fermenting Gram negative bacillus, $B$. cepacia was $100 \%$ resistant to all antimicrobial agents tested.

Interestingly, none of the tested drugs achieved sensitivity rates above $50 \%$ for $A$. baumannii and A. calcooceticus. Similar result was obtained in a study conducted by $\mathrm{Lu}$ et al [22], Sivaraman et al [26], Mostof et al [27], and Benachinmardi et al [28]. An inherent resistance resulting from the bacterial cell structure, together with a gradual acquisition of genetic determinants of resistance over time have been incriminated as cause of drug resistance development in the bacterium [18].

Changes in the susceptibility of Gram- positive cocci in hospital and community settings have been reported 
worldwide [29]. Similar to many earlier studies [4, 30, 31], the level of drug resistance of Gram-positive cocci to erythromycin $(60.4 \%)$, clindamycin $(52.2 \%)$, and tetracycline $(51.3 \%)$, the most commonly prescribed drugs in Ethiopia was very high. However, the overall drug sensitivity rates of Gram-positive bacterial isolates towards many antimicrobial categories such as lipopeptides (daptomycin), glycopeptides (vancomycin) oxazolidinones (linezolid), glycylcyclines (tigecycline), fluoroquinolones (ciprofloxacin, levofloxacin \& moxifloxacin), aminoglycosides (gentamycin) tetracycline (minocycline) and streptogramins (quinupristin-dalfopristin) were very high. The susceptibility rate of Gram positive bacteria extends from $75.3 \%$ for minocycline to $99.1 \%$ for vancomycin. Except one isolate of $S$. aureus (intermediate), all isolates of $S$. aureus and all isolates of CoagulaseNegative staphylococci were $100 \%$ susceptible to vancomycin. Our finding was in line with the findings of earlier studies [16, 32]. Tiemersma et al [32] analyzed 50,759 S. aureus isolates collected from 1999-2002 in 495 hospitals in 26 countries in Europe for their drug susceptibility profile and their result revealed that none of the isolates was resistant to vancomycin. However, vancomycin resistant coagulase-positive and Coagulase-Negative Staphylococci have been isolated in many countries. Studies conducted in Nigeria by Moses et al [33], in Bangladesh by Hasan et al [34], and in Ethiopia by Ten et al [35] reported 5.3\%, 4.2\% and $14 \%$ vancomycine resistant $S$. aureus, respectively. Similar studies conducted in Ethiopia by Ten et al [35] and Amare et al [36] documented a $13.4 \%$ and $4.5 \%$ prevalence rate of vancomycin resistant Coagulase -Negative Staphylococci. Tigecycline and linezolid as the most active antimicrobial agents against Gram-positive pathogens including enterococci, streptococci and staphylococci has been reported [37]. The relatively low level of resistance to these drugs may be, these drugs had been in the market for a relatively short period of time as compared to drugs such as tetracycline, amoxicillin and erythromycin [38]. In conclusion high culture positivity rate of wound infection was depicted. The resistance rates of bacterial isolates to the commonly prescribed drugs were very high.

\section{Conclusions}

The culture positivity rate recorded in the present study was compatible higher than similar studies conducted locally. Bacteria implicated in causing wound infection reported in this study were so diverse compared to previous local and international studies. High culture positive rate of wound infection and identification of bacteria that were not reported in similar studies warrants a continuous epidemiological survey of wound infection in health institutions across the country. The antimicrobial susceptibility pattern of bacteria against the commonly prescribed drugs such as erythromycin, tetracycline, ampicillin and amoxicillin demonstrated that these drugs would not cover the majority of bacterial wound infections as a single agent for empirical treatment. As the result, other alternatives should be considered.

\section{Strength sand imitations of the study}

Isolation and characterization of diverse bacterial species some of which have never been reported in previous studies, Identification of all bacteria associated with wound infection including Coagulase Negative staphylococcus to the species level, and testing the drug susceptibility of bacterial isolates against a large number of antimicrobial agents by automated methods were the strengths of this study over similar earlier studies. However, the study was not without limitations. Lack of information whether wound infections were hospital acquired or community-acquired and lack of information about the site of infection were major limitations. In addition to these, mechanisms of drug resistant development in our isolates were not studied as a result of problems associated with facilities. Instead, we have maintained all isolates in culture for further studies.

\section{Competing of Interests}

The work does not have no financial and/or non-financial competing interest. The authors declare that there is no conflict of interests regarding the publication of this paper. The study was approved by the Internal Review Board (IRB) of Arsho Advanced Medical Laboratory private limited company.

\section{Acknowledgements}

The authors would like to acknowledge Arsho Advanced medical Laboratory for the provision of laboratory supplies and allowing us to use the VITEK 2 compact system for free. The authors are also indebted to the patients.

\section{References}

[1] Esebelahie NO, Newton-Esebelahie FO, Omoregie R. Aerobic bacteria isolated from infected wound. Afr J Clin Exp Microbial. 2013; 14:1595-689.

[2] Bowler PG, Duerden BI, Armstrong DG. Wound microbiology and associated approaches to wound management. Clin Microbiol Rev. 2001; 14:244-269.

[3] Onderdonk AB. Pharmacodynamics and microbiology of tovrafloxacin in animal models of surgical infection. Am J Surg. 1998; 176:39S-45S.

[4] Lipsky BA, Weigelt JA, Gupta V, Killian A, Peng MM. Skin, soft tissue, bone, and joint infections in hospitalized patients: epidemiology and microbiological, clinical, and economic outcomes. Infect Control Hosp Epidemiol. 2007; 28:1290 1298.

[5] Zervos MJ, Freeman K, Haque N, Pokharna H, Raut M, Kim M. Epidemiology and outcomes of complicated skin and soft tissue infections in hospitalized patients. J Clin Microbiol. 2012; 50:238-245.

[6] Howell-Jones RS, Wilson MJ, Hill KE, Howard AJ, Price PE, Thomas DW. A review of the microbiology, antibiotic usage and resistance in chronic skin wounds. J Antimicrob Chemother. 2005; 55: 143-149. 
[7] Samuel SO, Kayode OO, Musa OI, Nwigwe GC, Abanerin AO. Nosocomial infections and the challenges of control in developing countries. Afr J Clin Exp Microbiol. 2010; 11:102-110.

[8] Sani PG, Kodwavwala MYD. Wound infections at the Kenyatta National Hospital. Pruc Assoc Surg East Afr 1991; 14: 36-38.

[9] De Macedo JLS, Santos JB. Bacterial and fungal colonization of burn wounds. Mem Inst Oswaldo Cruz. 2005; 100: 535539 .

[10] Nichols RE. Preventing surgical site infections: a surgeon's perspective Emerg Infect Dis. 2001; 7: 220-224.

[11] Bitew A, Molalign T, Chanie M. Species distribution and antibiotic susceptibility of bacterial uropathogens among patients complaining of urinary tract infections. BMC infectious disease 2017; 17:654. DOI 10.1186/s12879-0172743-8.

[12] Mulu A, Moges F, Tessema B, Kassu A. Pattern and multiple drug resistance of bacterial pathogens isolated from wound infection at University of Gondar Teaching Hospital, Northwest Ethiopia. Ethiop Med J. 2006; 44:251-31.

[13] Mulugeta KA. Bayeh AB. Bacteriology and antibiogram of pathogens from wound infections at Dessie Laboratory, North East Ethiopia. Tanzania J Health Res. 2011; 13: 1-10.

[14] Anguzu JR, Olila D. Drug sensitivity patterns of bacterial isolates from septic post-operative wounds in a regional referral hospital in Uganda. Afr Health Sci. 2007; 7: 148-154.

[15] Oladeinde BH, Omoregie R, Olley M, Anunibe JA, Onifade AA. A 5 - year surveillance of wound infections at a rural tertiary hospital in Nigeria. Afr Health Sci. 2013; 13: 351356.

[16] Mohammedaman M, Alemseged A, Tsegaye S. Antimicrobial susceptibility pattern of bacterial isolates from wound infection and their sensitivity to alternative topical agents at Jimma University Specialized Hospital, South-West Ethiopia. Annals of Clin Microbiol and Antimicrob. 2014; 13: 14-10.

[17] Giacometti A, Cirioni O, Schimizzi AM. Epidemiology and Microbiology of Surgical Wound Infections. J Clin Microbiol. 2000; 38: 918-922.

[18] Karlowsky JA, Draghi DC, Jones ME, Thornsberry C, Friedland IR, Sahm D. Surveillance for antimicrobial susceptibility among clinical isolates of Pseudomonas aeruginosa and Acinetobacter baumannii from hospitalized patients in the United States, 1998-2001. Antimicrob Agents Chemother. 2003; 47: 1681-1688.

[19] Scott P, Deye G, Srinivasan A, Murray C, Moran M, Hulten ED. An outbreak of multidrug-resistant Acinetobacter baumannii-calcoaceticus complex infection in the US military health care system associated with military operations in Iraq. Clin Infect Dis. 2007; 44:1577-1584.

[20] Mohammed A, Adeshina GO, Ibrahim, YK. Incidence and Antibiotic susceptibility pattern of bacterial isolates from wound infections in a Tertiary Hospital in Nigeria. Trop $\mathbf{J}$ Pharm Res. 2013; 12: 617-621.

[21] Manikandan C, Amsath A. Antibiotic susceptibility of bacterial strains isolated from wound infection patients in Pattukkottai, Tamilnadu, India. Int J Curr Microbiol App Sci. 2013. 2: 195-203.
[22] Lu PL, Liu YC, Toh HS, Lee YL, Liu YM, Ho CM et al. Epidemiology and antimicrobial susceptibility profiles of Gram-negative bacteria causing urinary tract infections in the Asia-Pacific region: 2009-2010 results from the Study for Monitoring Antimicrobial Resistance Trends (SMART). 2012; Int. J Antimicrob Agents 40S1: S37-S4322.

[23] Bours PHA, Polak R, Hoepelman AIM, Delgado E, Jarquin A, Matute AJ. Increasing resistance in community-acquired urinary tract infections in Latin America, five years after the implementation of national therapeutic guidelines. Int. J. Infect. Dis. 2010; 4: e770-e774.

[24] Mojtahedzadeh M, Panahi Y, Fazeli MR, Najafi A, Pazouki M, Navehsi BM. Intensive care unit-acquired urinary tract infections in patients admitted with sepsis: etiology, risk factors, and patterns of antimicrobial resistance. Int J Infect Dis. $2008 ; 12$ : $312-318$.

[25] Juyal D, Prakash R, Shanakarnarayan SA, Sharma M, Negi V, Sharma N. Prevalence of non-fermenting gram negative bacilli and their in vitro susceptibility pattern in a tertiary care hospital of Uttarakhand: A study from foothills of Himalayas. Saudi J.r Health Sci. 2013; 2: 108-112.

[26] Sivaraman V, Umadevi S, Srirangaraj S, Kali A, Seethaks. Multidrug resistant Acinetobacer species from various clinical samples in a tertiary care hospital from South India. Australasian Med. J. 2013:12:697-700.

[27] Mostof S, Mirnejad R, Faramaz M. Multi-drug resistance in Acinetobacter baumannii strains isolated from clinical specimens from three hospitals in Tehran-Iran. Afr. J Microbiol Res 2011; 5: 3579-82.

[28] Benachinmardi KK, Padmavathy M, Malini J, Naveneeth BV. Prevalence of non-fermenting Gram-negative bacilli and their in vitro susceptibility pattern at a tertiary care teaching hospital. J. Scientific Society. 214; 41:162-166.

[29] Orrett FA. Antimicrobial sensitivity patterns of aerobic bacterial blood isolates: experience at a University Hospital in Trinidad. Intl J Antimicrob. Agents. 2001; 17:75-77.

[30] Biadgelegne, F, Abera, B, Alem A, Anagaw B. Bacterial isolates from wound infection and their antimicrobial susceptibility pattern in Felege Hiwot Referral Hospital, Northwest Ethiopia. Ethiop J Health Sci. 2009; 19: 173-177.

[31] Petkovs`ek Z, Elers`ic`` K, Gubina M., Z` gur-Bertok D, Erjavec MS. Virulence Potential of $E$. coli isolates from skin and soft tissue infections. J Clin Microbiol. 2009; 47: 1811-1817.

[32] Tiemersma EW, Stef LAM, Bronzwaer, Lyytikäinen O, Degener JE, Schrijnemakers P. Methicillin-resistant $S$. aureus in Europe, 1999-2002. Emerg Infect Dis. 2004; 10: 1627-1634.

[33] Moses AA, Uchenna UA, Nworie O. Epidemiology of Vancomycin Resistant $S$. aureus among Clinical Isolates in a Tertiary Hospital in Abakaliki, Nigeria. Am J Epidemiol Infect Dis. 2013; 1: 24-26.

[34] Hasan R, Acharjee M, Noor R. Prevalence of vancomycin resistant $S$. aureus (VRSA) in methicillin resistant $S$. aureus (MRSA) strains isolated from burn wound infection. TzuChi Med J. 2016; 28: 49-53.

[35] Ten Hav R-J, Tesfaye M, ten Have WR, Nigussie M. Profiling of antibiotic resistance of bacterial species recovered from routine clinical isolates in Ethiopia. Ann Clin Microbial Antimicrob. 2017; 16:46 DOI 10 1136/s 1294-017-0221-1. 
[36] Amare B, Abdurrahman Z, Moges B, Ali J, Muluken L, Alemayehu M. Postoperative Surgical Site Bacterial Infections and Drug Susceptibility Patterns at Gondar University Teaching Hospital, Northwest Ethiopia. J Bacteriol Parasitol. 2011; 2-8. http://dx.doi.org/10.4172/21559597.1000126 .

[37] Balodea A, Volga Punda-Poli'c V, Dowzickyc JM. Antimicrobial susceptibility of Gram-negative and Gram- positive bacteria collected from countries in Eastern Europe: results from the Tigecycline Evaluation and Surveillance Trial (T.E.S.T.) 2004-2010. Int. J. Antimicrob.1 Agents. 2013; 41: $527-535$.

[38] Newman MJ, Enoch F, Asamoah-Adu A, Sampane-Donkor E. The Ghanaian- Dutch Collaboration for Health Research and Development Project Number 2001/GD/072006; Technical Repot Series No. 5. 